\title{
La technique sur le tas. Aspects sociaux de l'utilisation des machines sur les chantiers de construction (Suisse, fin XIX ${ }^{\mathrm{e}}$ siècle)
}

Techniques on the spot. Social features of the uses of machines on building sites (late $19^{\text {th }}$ Switzerland)

Technik auf der Baumstelle. Die Nutzung von Baumaschinen als soziales

Phänomen (Switzerland, 19. Jahrhunderts)

\section{Christoph Rauhut}

Traducteur : Manuel Charpy et François Jarrige

\section{Q OpenEdition}

\section{Journals}

Édition électronique

URL : http://journals.openedition.org/rh19/4344

DOl : $10.4000 /$ rh 19.4344

ISSN : $1777-5329$

\section{Éditeur}

La Société de 1848

\section{Édition imprimée}

Date de publication : 31 décembre 2012

Pagination : 127-142

ISSN : 1265-1354

\section{Référence électronique}

Christoph Rauhut, «La technique sur le tas. Aspects sociaux de l'utilisation des machines sur les chantiers de construction (Suisse, fin XIXe siècle) ", Revue d'histoire du XIXe siècle [En ligne], 45 | 2012, mis en ligne le 31 décembre 2015, consulté le 01 mai 2019. URL : http://journals.openedition.org/ rh19/4344; DOI : 10.4000/rh19.4344 


\section{CHRISTOPH RAUHUT}

\section{La technique sur le tas. Aspects sociaux de l'utilisation des machines sur les chantiers de construction (Suisse, fin XIXe siècle)}

Le 8 février 1868, l'inventeur E. R. Gard, de Chicago, lance un défi à ses concurrents en annonçant publiquement que sa «machine perfectionnée à fabriquer les briques est la meilleure du monde ${ }^{1}$. Pour étayer cette affirmation, il propose de lancer un concours qui est à l'origine de la "controverse des machines à fabriquer les briques» et fait l'objet d'une publication presque quotidienne dans The Chicago Tribune pendant les trois semaines qui suivent l'annonce $^{2}$. La controverse porte principalement sur les critères à retenir pour juger du mérite respectif des différents procédés : faut-il retenir la quantité ou bien la qualité de la production? D'un côté, on trouve les inventeurs qui vantent l'efficacité de leurs nouvelles machines mues par la vapeur, de l'autre les briquetiers qui défendent les procédés en usage en affirmant que la dégradation de la qualité, qui ne manquerait pas d'accompagner l'utilisation des nouvelles machines, les obligerait à produire encore plus et donc à solliciter davantage les anciens procédés. Près de dix ans plus tôt, un débat similaire avait eu lieu à Londres à propos de la «fabrication des briques au moyen des machines ${ }^{3}$. Deux groupes s'affrontaient déjà à cette occasion : les inventeurs s'opposaient aux fabricants de briques hostiles par principe aux machines ${ }^{4}$. Les fabricants de briques dénonçaient «les fréquents échecs pratiques» des machines, ils mettaient en avant la qualité de leur travail produit par la supériorité de leurs savoirs et de leur expertise 5 . À première vue,

1. E.R. Gard, 'A Challenge', Chicago Tribune, le 8 février 1868, p. 4.

2. A. J. Knisely, "Brick-laying - Mr. Gard's challenge", Chicago Tribune, 13 février 1868, p. 3; E. R. Gard, "Brick-laying - Mr. Gard's challenge - Answer to A. J. K.", Chicago Tribune, 14 février 1868, p. 4; E. R. Gard, "The brick-machine controversy", Chicago Tribune, 20 février 1868, p. 4; A. J. Knisely, "The brick-machine controversy - Conscience on a bender", Chicago Tribune, 21 février 1868, p. 4; E. R. Gard, "Mr. Gard's challenge", Chicago Tribune, 22 février 1868, p. 4; A. J. Knisely, "The brickmachine controversy", Chicago Tribune, 27 février 1868, p. 4; O. B. Gruen, "The brick-machine controversy", Chicago Tribune, 29 février 1868, p. 4.

3. Humphre Chamberlain, "The Manufacture of Bricks by Machinery", Journal of the Society of Arts, IV, 185, 1856, p. 491-501.

4. Idem, p. 499.

5. Idem, p. 493. 
ces débats peuvent sembler relever de questions essentiellement techniques, mais ils s'enracinent en réalité dans des logiques sociales : il est évident que l'expérience professionnelle et sociale des divers protagonistes a largement déterminé leurs positions à l'égard des machines. Ces deux controverses sont exemplaires des débats que soulève la mécanisation dans l'industrie du bâtiment au XIX ${ }^{e}$ siècle. Trois types d'acteurs s'affrontent : les "habiles» ingénieurs mécaniciens, les entrepreneurs qui suivent les logiques du marché, et les artisans des métiers produisant de façon traditionnelle briques et mortiers - ou pour le dire de façon plus abstraite, le développement technologique, les usages potentiels et les usages réels.

En partant de ces deux affaires, l'article qui suit cherche à examiner de façon approfondie l'utilisation quotidienne des machines de construction au XIX ${ }^{\mathrm{e}}$ siècle. Celles-ci peuvent être définies dans ce contexte comme des objets techniques utilisés sur les chantiers pour diminuer le travail manuel, à l'exclusion bien entendu des outils des artisans ${ }^{6}$. Ces machines de construction comprennent, par exemple, des équipements tels que des crics de levage, des palans, des grues, des pompes et des pilonneuses. Les terrains explorés sont les chantiers de construction les plus répandus à la fin du XIX ${ }^{\mathrm{e}}$ siècle : immeubles d'habitation, écoles, bureaux, églises, etc. Ces chantiers typiques de la ville de cette période ont pourtant été largement négligés par le grand récit héroïque habituel de l'historiographie de la construction qui s'est surtout intéressé aux réalisations les plus vastes et les plus spectaculaires ${ }^{7}$. Le grand nombre de groupes différents présents sur ces sites offre pourtant l'opportunité d'examiner les multiples interactions à l'œuvre sur les chantiers et la façon dont elles different d'un groupe à l'autre. Au tournant du siècle, les artisans traditionnels continuent de travailler au même endroit que les nouveaux spécialistes des entreprises récemment fondées. Les techniques de construction anciennes et éprouvées côtoient donc les nouveaux procédés de l'industrie ${ }^{8}$. Cette coexistence est l'occasion d'observer les motivations qui justifient l'emploi ou, à l'inverse, le refus d'utiliser les machines de construction. Ce refus ne doit pas être interprété comme une manifestation de technophobie, mais plutôt comme le résultat d'une façon différente de comprendre et d'envisager ce qu'est l'outil ou la technique la plus utile? ${ }^{9}$. En

6. La définition exacte de l'expression «machine de construction" [Baumaschine] est l'enjeu de discussion au XIX e siècle. La définition retenue ici résume les usages les plus fréquents dans le secteur des travaux publics de l'époque. Les ouvriers de la construction utilisaient souvent l'expression «équipement de construction" [Baugeräte].

7. Voir Tom F. Peters, Building the Nineteenth Century, Cambridge, Massachusetts, MIT Press, 1996; Hans Straub, Die Geschichte der Bauingenieurkunst, Basel, Birkhäuser, 1949.

8. Christoph Rauhut, „Die Baustelle um 1900 - Materialvielfalt, Konstruktionspluralismus, Akteurspluralismus", in Uta Hassler et Christoph Rauhut (ed.), Bautechnik des Historismus, München, Hirmer, 2012, p. 262-279.

9. Marcus Popplow, „Neu, nützlich und erfindungsreich. Die Ingenieure der Reanissance als Schrittmacher der modernen Deutung von Technik", in Gisela Engel et Nicole C. Karafyllis (ed.), Technik in der Frühen Neuzeit - Schrittmacher der europäischen Moderne, Frankfurt am Main, Vittorio Klostermann, 2004, p. 336-355, ici p. 338. 
examinant de près les différents groupes sociaux présents sur le chantier, on tâchera donc d'identifier les raisons qui justifient ces interprétations différentes de ce qu'est la technique la plus efficace.

De nombreux acteurs sont impliqués dans la conception et l'utilisation des machines de construction sur les chantiers : on trouve des ingénieurs mécaniciens, qui conçoivent les machines; des négociants locaux, qui les vendent ou les louent; et enfin ceux qui les actionnent sur le chantier. Ce dernier groupe peut lui-même être subdivisé en plusieurs sous-ensembles : les entrepreneurs qui veulent ou qui sont contraints d'utiliser une machine; les contremaitres qui supervisent le travail et l'utilisation des engins; et enfin les ouvriers qui actionnent et travaillent avec ces machines. C'est en comparant les opinions émises par les ingénieurs, les vendeurs de machines et les acteurs présents sur le chantier que l'on peut approcher les différences qui existent entre, d'une part, le développement technique et le fait de posséder les machines - confondu souvent avec l'usage -, les usages possibles permis par les machines d'autre part, et, enfin, les usages réels au quotidien. C'est de même en comparant les attitudes et les savoirs à l'égard des machines chez les entrepreneurs, les contremaîtres et les ouvriers que l'on peut éclairer l'utilisation ou le refus d'utiliser les artefacts techniques, en lien avec l'origine sociale de chacun des protagonistes. L'expression "origine sociale" peut être définie, dans ce contexte, comme participant d'une culture professionnelle, mais aussi comme le fruit de l'éducation et des savoirs que possède un individu - cet article interrogeant ainsi les formes de l'expertise. Ce travail repose sur une série d'enquêtes menées sur divers cas de chantiers de construction dans la région de Zurich, en Suisse. L'enjeu est de saisir de quelle manière l'organisation sociale du chantier a été déstabilisée par l'arrivée des machines, mais aussi les transformations dans l'organisation du travail, comme dans la répartition des responsabilités et des compétences qu'elle a pu générer.

\section{Améliorations "héroÏQues", fourniture locale ET DEMANDE SUR LES CHANTIERS}

Pour éclairer le fossé qui existe entre le développement technologique et les pratiques effectives des machines de construction, au regard des différentes sphères sociales, on partira d'une comparaison entre deux sources : d'une part le Handbuch der Ingenieurwissenschaften, un manuel abrégé de science de l'ingénieur publié entre 1880 et les années 1920; et d'autre part le Schweizer Bau- \& Ingenieur-Kalender, une synthèse publiée chaque année et comprenant les prix pratiqués dans le secteur du bâtiment en Suisse. Ces deux sources imprimées comportent des développements sur les machines de construction : la quatrième partie du Handbuch der Ingenieurwissenschaften 
leur est spécifiquement consacrée ${ }^{10}$; de plus, il comprend un chapitre entier dédié aux machines utilisées sur les chantiers ordinaires qui constituent le terrain d'enquête privilégié de ce travail ${ }^{11}$. De même le Schweizer Bau- \& Ingenieur-Kalender comprend aussi un chapitre spécifique sur le sujet.

La juxtaposition de ces deux sources ne sera pas envisagée simplement comme une comparaison entre deux discours, mais plutôt comme le témoignage du fossé qui sépare deux groupes sociaux ${ }^{12}$. Le Handbuch der Ingenieurwissenschaften est en effet un abrégé écrit par et pour une "élite technicienne». L'un des principaux initiateurs de la série est l'ingénieur des chemins de fer Edmund Heusinger von Waldegg; parmi les principaux auteurs de la partie dédiée aux machines de construction, on trouve aussi $\mathrm{F}$. Lincke, H. Weihe et L. von Willmann. Ce dernier est également en charge de la partie consacrée aux machines utilisées sur les chantiers ordinaires. Ces auteurs ont des parcours biographiques assez semblables et ils ont suivi une éducation identique. Ils sortent tous des nouvelles écoles polytechniques fondées dans les États allemands, ils y enseignent comme professeur, et ils ont d'ailleurs tous été professeurs au Technische Hochschule zu Darmstadt.

Le Handbuch der Ingenieurwissenschaften vise le même public. Il a été conçu comme un aperçu des "machines nécessaires à la construction, basé sur une approche scientifique, et en vue d'être utile dans les écoles comme dans la pratique $»^{13}$ (fig. 1). De même que les auteurs doivent être réinscrits dans la culture polytechnicienne de l'époque, il en va de même du livre; cette mentalité considère la science de l'ingénieur comme la combinaison d'un ensemble de savoirs scientifiques académiques et de savoir-faire empiriques ${ }^{14}$. Dans le cadre de la question soulevée, deux aspects semblent importants. Tout d'abord, le livre illustre l'attitude du groupe des "polytechniciens» à l'égard des machines de construction, attitude manifeste dès la préface : «On a très rarement observé une utilisation rationnelle des inventions du génie mécanique, en fait il existe une très forte et fréquente adhésion à l'égard des machines et des méthodes primitives, anciennes et couteuses» ${ }^{15}$. Les

10. L. Franzius et F. Lincke (ed.), Die Baumaschinen, Handbuch der Ingenieurwissenschaften 4, 3 volumes, Leipzig, Verlag von Wilhelm Engelmann, 1883 -1890 (2 ème édition, 4 volumes, 1897 -1911).

11. Pröll, Scharowsky et L. von Willmann, «Hilfsanlagen für den Materialtransport und die Errichtung von Hochbauten ", in L. Franzius et F. Lincke (ed.), Die Baumaschinen... op. cit., volume 2, 1885, p. $1-62$.

12. Rapprocher des publications et leur contenu de divers univers sociaux constitue une approche fréquente et répandue en histoire des sciences : voir Christel Meier, „Der Wandel der Enzyklopädie des Mittelalters vom Weltbuch zum Thesaurus sozial gebundenen Kulturwissens : am Beispiel der Artes mechanica“", in Franz M. Eybl et al. (ed.), Enzykolpädien der Frühen Neuzeit, Tübingen, Max Niemeyer Verlag, 1995, p. 19-42.

13. „Soll in wissenschaftlicher Darstellung eine für Studium und Praxis dienlichen Übersicht der zum Bauen erforderlichen Maschinen geben“, L. Franzius et F. Lincke (ed.), Die Baumaschinen... op. cit., volume 1,1883 , p. v.

14. Torsten Meyer, 'The "Science of Building" as Polytechnic Discipline in the $19^{\text {th }}$ Century', in Robert Carvais, André Guillerme, Valérie Nègre [dir.], Nuts \& Bolts of Construction. History. Culture, Technology and Society, 3 volumes, Paris, Picard, 2012, volume 1, p. 19-25.

15. „Eine rationelle Benutzung des durch die Entwicklung des Maschinenbaues Gebotenen [hat] so 


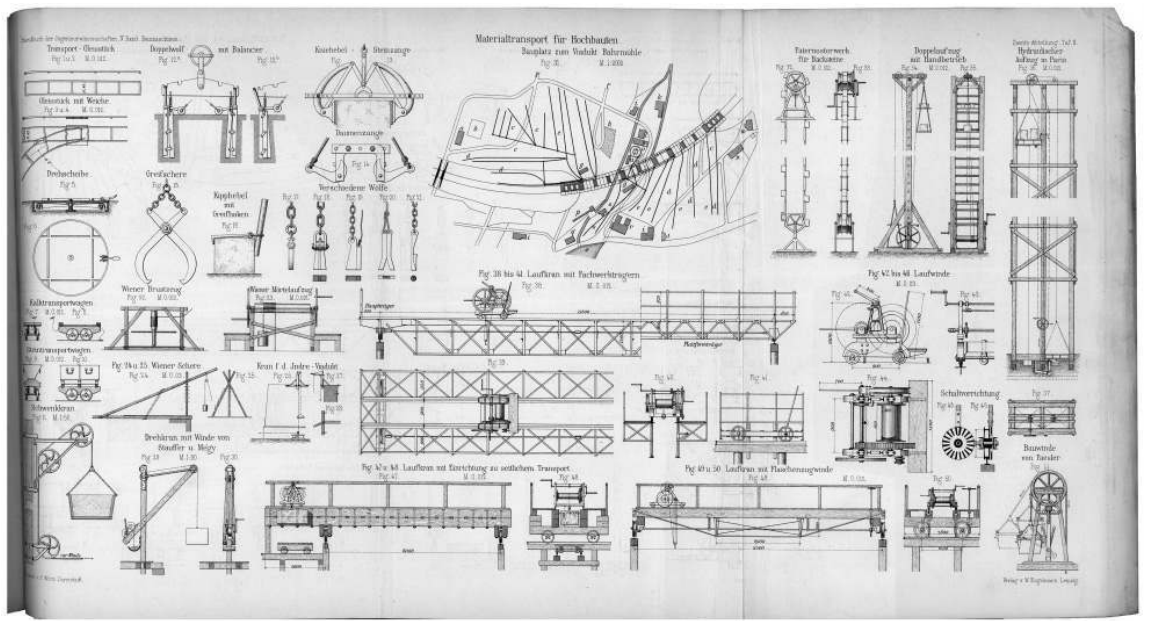

Fig. 1. Exemple d'une machine de construction pour le transport dans L. Franzius et F. Lincke (ed.), Die Baumaschinen, Handbuch der Ingenieurwissenschaften, 4, volume 2, Leipzig, Verlag von Wilhelm Engelmann, 1885, plate 1.

nouvelles machines perfectionnées mises au point par les ingénieurs sont clairement, pour eux, l'équipement le plus approprié. En second lieu, le but du Handbuch der Ingenieurwissenschaften est de représenter tout le champ de la construction, dans sa globalité comme dans les détails ${ }^{16}$. Par conséquent, le livre offre un aperçu de presque toutes les machines connues à l'époque et dessine ainsi un champ abstrait de technologies.

Par contraste, le Schweizer Bau- \& Ingenieur-Kalender décrit le champ des usages possibles. Ce calendrier annuel a été publié pour la première fois en 1880 sous le titre de Taschenbuch der schweizerischen Baupreise (Manuel des prix de construction) par l'architecte Alexander Koch ${ }^{17}$, l'un des principaux architectes de Zurich qui cherche à rendre le secteur de la construction à la fois plus professionnel et plus intelligible ${ }^{18}$. Son objectif est d'offrir un aperçu des prix des matériaux et des équipements de construction en Suisse, et particulièrement dans la région de Zurich qui était alors la capitale économique et culturelle de la Suisse germanophone. Les données présentées dans le Schweizer Bau- \& Ingenieur-Kalender sont élaborées grâce aux observations recueillies auprès des revendeurs locaux des matériaux de construction, les références ainsi fournies nous permettent de retracer leurs contributions. Le

selten gefunden, vielmehr [wird] so oft und so hartnäckig noch an der Verwendung primitiver, veralteter und kostspieliger Hülfsmittel und Methoden festgehalten“, L. Franzius et F. Lincke (ed.), Die Baumaschinen... op. cit., volume 1, 1883, p. vI.

16. Idem.

17. Alexander Koch (ed.), Taschenbuch der Schweizerischen Baupreise, Zürich, Cäsar Schmidt, 1880. Dans les années qui suivent, on trouve un grand nombre de versions différentes de ce titre, pour simplifier nous n'emploierons que le nom le plus fréquemment utilisé de Schweizer Bau- \& Ingenieur-Kalender. 18. Christoph Rauhut, „Das tragische Genie. Zum 100. Todestag des Architekten Alexander Koch - eine aussergewöhnliche Zürcher Persönlichkeit“, Neue Zürcher Zeitung, le 28 avril 2011. 


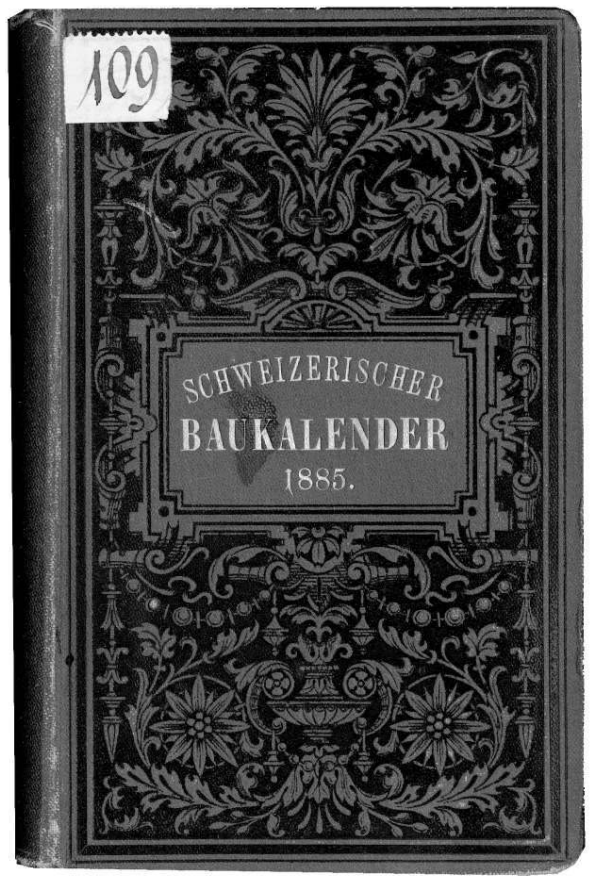

Fig. 2. Couverture du Schweizerischer Baukalender (Alexander Koch (ed.), Schweizerischer Baukalender, Zürich, Cäsar Schmidt, 1885).

Kalender peut donc être considéré comme un reflet assez fidèle de la situation du marché local, c'est-à-dire des utilisations possibles.

En outre, le Schweizer Bau- \& Ingenieur-Kalender peut être relié à un groupe plus large. Cette publication s'adresse en effet aux architectes, directement impliqués dans la pratique, et aux propriétaires des entreprises de construction, c'est-à-dire à ceux qui, dans le secteur de la construction, avaient à décider quel type de matériaux devait être utilisé et quel type de machine devait être employé. S'il n'est pas possible de définir ce groupe aussi précisément que celui des "polytechniciens", on peut néanmoins distinguer quelques-unes de ses caractéristiques. Si les architectes et les entrepreneurs travaillent bien dans le bâtiment, en revanche, ils ne sont pas physiquement présents sur les chantiers, ils appartiennent au groupe des décideurs; leurs connaissances émanent surtout de l'expérience acquise au cours de leurs travaux antérieurs, leur culture professionnelle est avant tout dirigée vers la satisfaction des exigences du marché. Ils lisent le Schweizer Bau- \& IngenieurKalender, non pas pour connaître les dernières innovations, mais d'abord pour savoir quelles sont les machines effectivement disponibles.

Dès lors, la comparaison des machines de construction qu'on trouve dans le Kalender et dans le Handbuch n'est pas seulement une comparaison entre deux sources, c'est surtout la comparaison entre deux groupes déjà évoqués dans l'introduction : d'un côté l'ingénieur mécanicien, de l'autre l'entrepreneur qui suit les dynamiques du marché. 


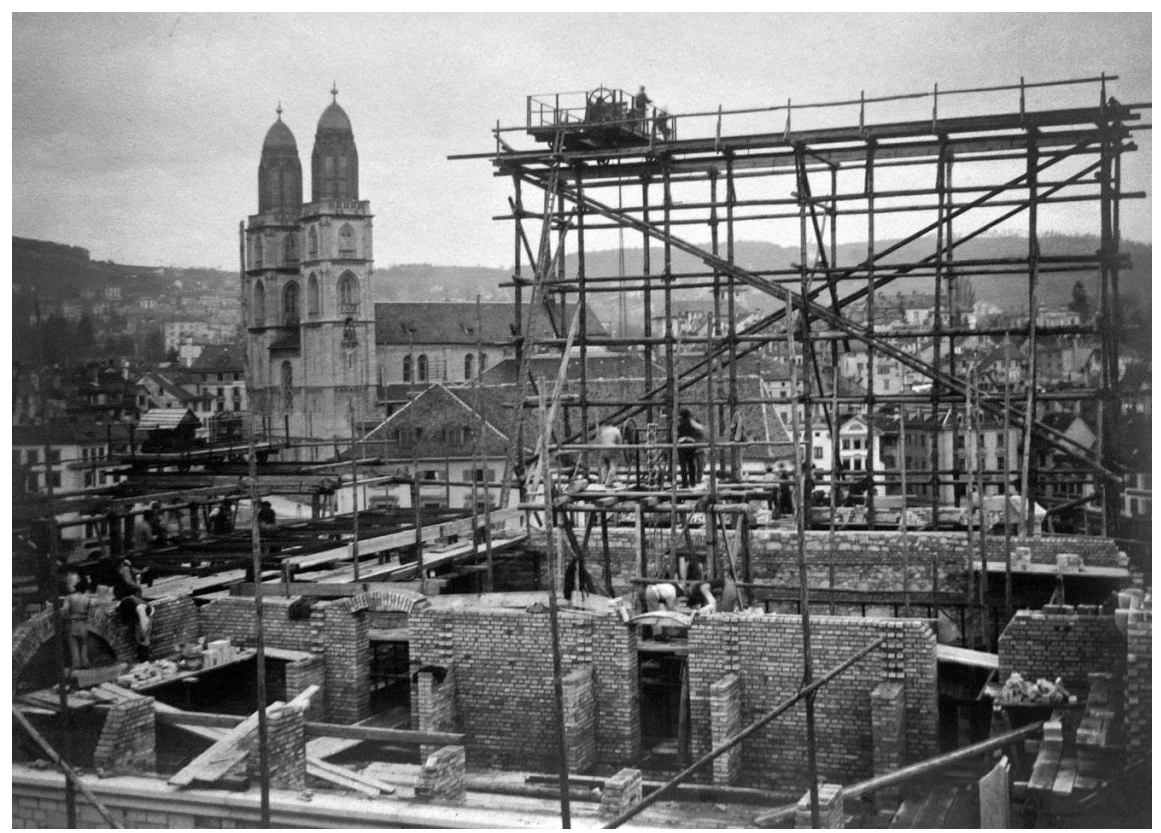

Fig. 3. Chantier de l'immeuble de bureaux 'Fraumünsteramt', Zürich, 1899. (Zentralbibliothek der Stadt Zürich, Graphische Sammlung, succession Robert Breitinger).

La comparaison des machines effectivement présentées dans ces publications valide ce constat : alors que le Handbuch couvre presque toute la gamme des machines de construction disponibles, le Kalender présente d'abord celles qui apparaissent utiles au regard de la situation du marché. Le chapitre consacré aux machines de construction pour les chantiers les plus courants dans le Handbuch peut ainsi se lire comme une chronique de tout ce qui avait été conçu à l'époque. Il s'étend sur un peu plus de soixante pages, avec autant de machines présentées. On peut également remarquer que pour chaque type de machine, on trouve la mention de plusieurs marques ${ }^{19}$. À l'inverse, dans son édition pourtant assez tardive de 1889, le Schweizer Bau- \& Ingenieur-Kalender ne présente que des machines de construction ayant un intérêt suffisamment grand pour faire l'objet d'un chapitre séparé, et seules trois pages leur sont consacrées. Par ailleurs, il s'agit de techniques très basiques : pompes, crics de levage et palans ${ }^{20}$. Même dans les éditions ultérieures, les descriptions des machines de construction complexes demeurent rares; la première mention d'une grue pivotante n'apparaît par exemple que dans l'édition de $1913^{21}$. Il existe ainsi un très clair décalage entre les types de procédés techniques pré-

19. La section portant sur les grues dans l'édition de 1885 comprend ainsi un modèle de la marque Satufer \& Méguy, une de Ph. Holzmann \& Co, une de Bebr. Weissmüller, une autre de Florio \& Co, etc. Pröll, Scharowsky et L. von Wilmmann, „Hilfsanlagen für den Materialtransport...“, op. cit., p. 28-31. 20. Martin Koch (ed.), Schweizerische Baukalender, Zürich, Cäsar Schmidt, 1889, p. 144-146.

21. Schweizer Baukalender, volume 2, Zürich, Cäsar Schmidt, 1913, p. 393. 
sentés dans les deux sources. Non seulement le nombre de machines décrites dans le Handbuch est beaucoup plus grand, mais par ailleurs le Kalender tend à privilégier les procédés les plus simples et basiques.

Cette différence n'est pas seulement le fruit des limites théoriques comme la langue, les conditions économiques, ou la distance géographique - qui influencent toujours le transfert des savoirs; elle est d'abord déterminée par des enjeux sociaux. Chaque groupe élabore en effet des critères distincts pour juger du bien fondé de l'utilisation des machines, en fonction de ses origines, de sa position singulière dans le processus de décision, mais aussi de ses connaissances et expériences propres sur le chantier. Les recherches récentes sur la construction sociale des technologies et des techniques ont recours aux facteurs économiques, politiques, aux ressources culturelles et aux possibilités techniques pour définir les "caractéristiques structurantes qui peuvent permettre de définir un groupe social pertinent $»^{22}$. Utiliser cette définition invite à inclure une plus grande diversité de groupes sociaux, soit tous les gens présents sur le site de construction. De ce fait, cela revient à questionner, outre le développement technologique et les usages potentiels des techniques, leurs usages réels sur le tas. Alors que les recherches sur la construction sociale de la technologie focalisent sur la distorsion entre promoteurs et usagers ${ }^{23}$, une analyse différenciée de chaque groupe apparait nécessaire, notamment dans le secteur de la construction, analyse sans laquelle il est impossible de saisir l'ensemble des groupes sociaux impliqués et leur rôle respectif.

\section{Appropriations et REJETS DES MaChines :}

ENTRE EXPERTISE ET PRATIQUES SOCIALES

Pour pouvoir discuter de l'usage effectif et de l'attitude vis-à-vis des machines en fonction de la position de chaque groupe sur les chantiers, il est nécessaire de s'arrêter sur des études de cas. Dans le cas de Zurich, un grand nombre de chantiers ont laissé des archives. Ces sources sont constituées des documents produits quotidiennement : archives personnelles des superviseurs des sites, contrats entre les autorités et les entreprises, et plus marginalement, les comptabilités, les traces matérielles et les photographies des chantiers ${ }^{24}$. Un des cas bien documentés concerne le chantier du bâtiment administratif du 'Fraumünsteramt' érigés entre 1898 et 1901 (fig. 3) 25 .

22. Voir Hans K. Klein et Daniel Lee Kleinmann, "The Social Construction of Technology : Structural Considerations", Science, Technology \& Human Values, XXVII, 1 (2002), p. 28-52, ici p. 43.

23. Idem, p. 40.

24. L'œuvre photographique de Robert Breitiger par exemple, un photographe amateur de l'époque, est conservée dans la principale bibliothèque de Zurich. Il s'est particulièrement intéressé aux objets techniques, notamment ceux qu'on trouvait sur les chantiers de construction. Zentralbibliothek der Stadt Zürich, Graphische Sammlung (ZB GS), succession Robert Breitinger.

25. Les dossiers dans les Archives municipales de Zurich (AdZ) sont : V.G.b.253; V.G.c.62; V.G.a.12; V.G.c.31 Série 1. Dessins et plans sont dans les archives „Amt für Hochbauten der Stadt Zürich“, 


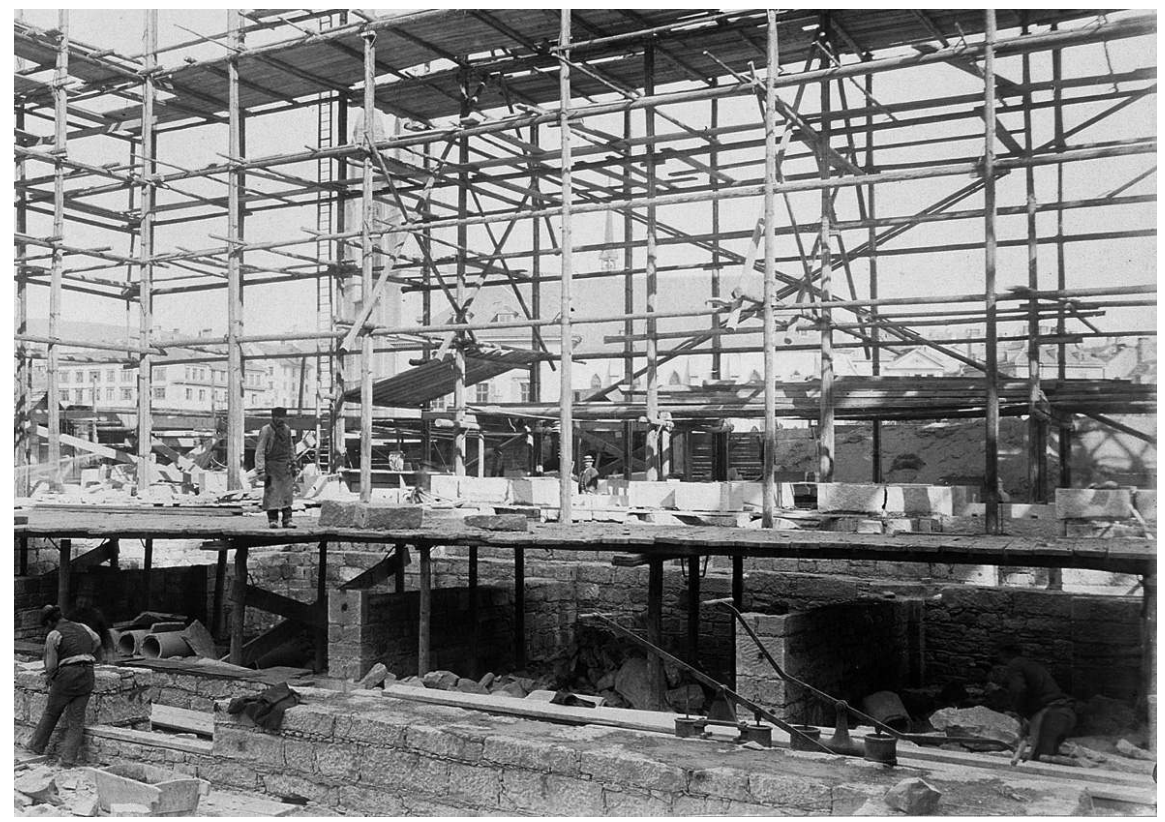

Fig. 4. Usage d'une pompe aspirante sur le chantier, chantier de l'immeuble de bureaux 'Fraumünsteramt', Zürich, 1899.

(Zentralbibliothek der Stadt Zürich, Graphische Sammlung, succession Robert Breitinger).

Le chantier est typique pour l'époque : on y trouve une large variété de matériaux, de dispositifs techniques ainsi que différents groupes professionnels ${ }^{26}$.

L'usage pratique des machines peut y être observé en s'arrêtant par exemple sur les efforts menés pour pomper les eaux résurgentes sur le chan$\operatorname{tie}^{27}$. Cette question qui mobilise différents groupes professionnels, avec des identités distinctes, et des positions sociales variables est exemplaire de ce qui se joue sur les chantiers.

Pomper les eaux souterraines pour rendre possible le travail de crépi dans les sous-sols des constructions est alors une pratique courante. Le contrat entre les autorités et l'entreprise de maçonnerie qui a en charge les travaux de crépi établit que le pompage est de la responsabilité de l'administration. Par conséquent, les premiers pompages entrepris sont initiés et dirigés par le superviseur du site, Gustav Kruck, qui représente les autorités compétentes sur le chantier. Tout au long du XIX $\mathrm{X}^{\mathrm{e}}$ siècle, la supervision du site devient une tâche centrale du fait de la complexification nouvelle des processus de

„Baugeschichtliches Archiv der Stadt Zürich“ und in the archive „Institut für Geschichte und Theorie der Architektur an der ETH Zürich". Les publications sur l'immeuble de bureaux Fraumünsteramt sont notamment : „Der Ausbau des Fraumünsteramtes in Zürich“, Schweizerische Bauzeitung, XXXI, 23 (1898), p. 169-171 ; „Das Städtische Verwaltungsgebäude im Fraumünsteramte in Zürich“, Schweizerische Bauzeitung, XLII, 1 (1903), p. 1-7; 2, p. 16-19; 4, p. 46-50.

26. Christoph Rauhut, Die Baustelle um 1900..., op. cit.

27. AdZ, V.G.b.253. : 2-4, notations du 28 mars au 27 avril 1899. 
construction. Elle est d'autant plus importante qu'elle est aussi le lieu de la rationalisation de ces processus ${ }^{28}$.

Les superviseurs du site sont généralement des artisans qualifiés qui se sont élevés socialement en suivant les cours des lycées techniques - dans le cas de la Suisse, le premier Technikum à Winterthur récemment fondé en 1874. Gustav Kruck incarne ce groupe professionnel du fait de son parcours et de son héritage social ${ }^{29}$. Dans le cas de ce chantier, il décide d'user de deux pompes aspirantes actionnées par la force physique de quatre manouvriers (fig. 4). Ces types de pompes avaient déjà été utilisés en de nombreuses occasions sur le chantier ${ }^{30}$. Il semble que ce choix relève de la culture et des habitudes professionnelles de Kruck qui choisit ce type de technique sans consulter au préalable les autres professionnels.

Le 28 mars 1899, les pompes sont mises en route. Dès la fin du jour, Kruck réalise que les résultats sont décevants, voire médiocres. Il écrit : "Dans les caves du Fraumünsterstrasse, les eaux de la nappe phréatique n’ont pas disparu $»^{31}$. Mais il décide de continuer même si, comme il le mentionne, c'est une méthode très coûteuse. Après deux jours de pompage en continu et de tentatives infructueuses, l'architecte Gustav Gull décide d'arrêter les pompes qui se révèlent inefficaces ${ }^{32}$.

Gull qui est l'autorité supérieure par rapport au directeur des travaux („Bauführer“) Kruck recommande les pompes à jets d'eau : «M. Gull ne veut pas continuer à utiliser les pompes. Il a l'intention d'utiliser la pompe à jets d'eau („Wasserstrahlpumpen“)»(fig. 5) ${ }^{33}$. Celle-ci, mise au point à l'origine pour l'industrie minière, connaît plusieurs versions dans le dernier quart du $\mathrm{XIX}^{\mathrm{e}}$ siècle développées par des producteurs de machines pour les chantiers, à l'instar de la 'Nagel'sche Strahlpump'34.

C'est sans surprise que Gustav Gull recommande ce type de pompe. Dans la littérature de l'époque dédiée aux architectes diplômés, on peut trouver de nombreuses références à ce genre d'équipements ${ }^{35}$ alors que dans le même

28. Christoph Rauhut, „'denn praktisch zu sein, liegt im Streben unserer Zeit' - Rationalisierungsbestrebungen auf der Baustelle um 1900“, in L. W. L.-Freilichtmuseum Hagen (ed.), Rationalisierung in Handwerksberufen. Beiträge des XXX. Gesprächskreis für Technikgeschichte. Tagung vom 2. bis 4. Juni 2011 im LWL-Freilichtmuseum Hagen, Hagen, 2012, p. 110-121.

29. Kruck n'a pas reçu d'éducation dans un "Technikum", mais a expérimenté une éducation comparable comme membre des services des travaux publics; voir Cristina Gutbrod, Gustav Gull (1858 -1942) - Architekt der Stadt Zürich 1890 -1911. Zwischen Vision und Baupolitik, thèse de doctorat, Zürich, 2009, p. 156.

30. Des photographies plus anciennes en témoignent, voir ZB GS, succession Robert Breitinger.

31. „Im Kübelraum Fraumünsterstrasse geht das Grundwasser nicht weg.“ AdZ, V.G.b.253.:2-4, notation du 30 mars 1899.

32. cf. Cristina Gutbrod, Gustav Gull..., op. cit.

33. „Herr Gull will deshalb nicht weiterfahren mit den Pumpen. Es soll vielleicht ein Versuch mit einer Injecoreinrichtung gemacht werden." AdZ, V.G.b.253.:2-4, notation du 30 mars 1899.

34. cf. Otto Lueger, Lexikon der gesamten Technik und ihrer Hilfswissenschaften, Stuttgart/Leipzig, Deutsche Verlags-Anstalt, 1904-10 (1 ére édition 1894-1899) 8 volumes, volume 8, p. 339.

35. Voir Kämp, „Nagel's Wasser-Saugapparat zum Entleeren von Baugruben“, Schweizerische Polytechnische Zeitschrift, X, 5 (1865), p. 149-152. 

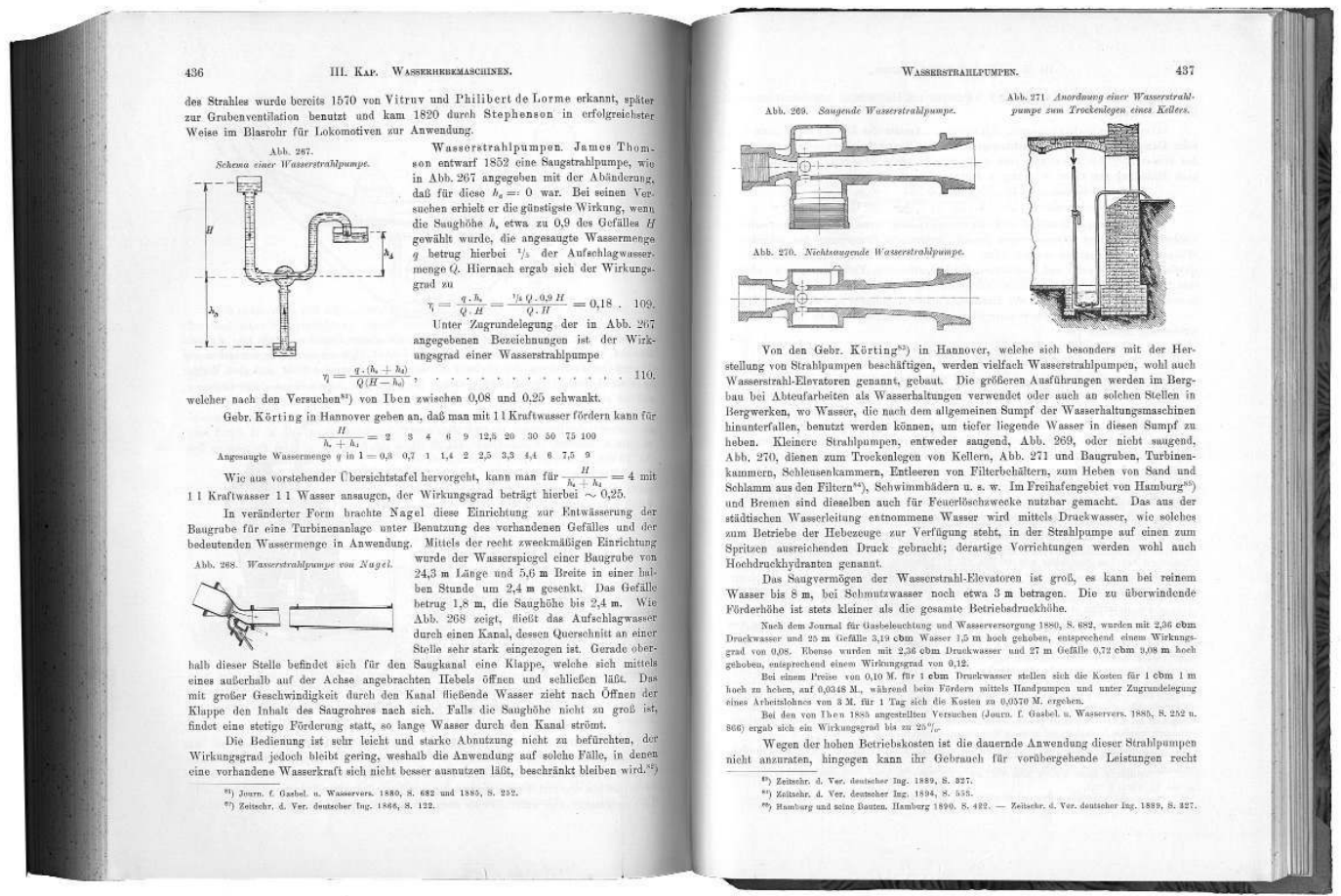

Fig. 5. Pages sur les pompes à jets d'eau tirés du Handbuch der Ingenieurwissenschaften

(L. Franzius et F. Lincke (ed.), Die Baumaschinen, Handbuch der Ingenieurwissenschaften 4, volume 4, Leipzig, Verlag von Wilhelm Engelmann, 3e édition 1910, p. 436-437).

temps les manuels pour superviseurs ne les mentionnent pas ou très rarement $^{36}$. Les commentaires rédigés par le superviseur Kruck montrent qu’il ignore à peu près tout de ce type de pompe ${ }^{37}$.

Nous ne savons pas quelles étaient les connaissances de Gull en matière de pompes mais son choix peut être mis en relation avec sa culture professionnelle et sa formation. Dans sa note de recommandation, il mentionne un objet technique dont les sources d'information à destination des architectes, et en particulier les journaux, font la publicité ${ }^{38}$. Plus encore, cette machine originale et rare peut être liée à la foi dans le progrès qui caractérise alors

36. Ils ne sont pas mentionnés par exemple dans les ouvrages tels que Gustav Tolkmitt, Bauaufsicht und Baufiuhrung, Berlin, Verlag von Wilhelm Ernst \& Sohn, 1899.

37. Il note: «Dans la matinée, un voyageur est venu, déclarant qu'il avait une pompe à jets d'eau qui pouvait faire mille litres par minute». („Am Abend ist ein Reisender hier, der behauptet Injectoren zu haben, welche pro. Mte. 1000 Liter Wasser entziehen") AdZ, V.G.b.253.:2-4, notation du 7 avril 1899. Non seulement il apparait étrange de prendre conseil auprès d'une personne de passage, d'autant qu'une telle pompe nécessiterait un débit de 4000 litres/minutes pour fonctionner, ce qui est bien au-delà de ce que peut fournir le système public de distribution.

38. Voir par exemple J. Körting, „Strahlpumpen“, Zeitschrift des Vereins deutscher Ingenieure, XXXIII, 1889, p. 327. On peut trouver de nombreuses publicités dans les journaux tels que Schweizerische Bauzeitung. 


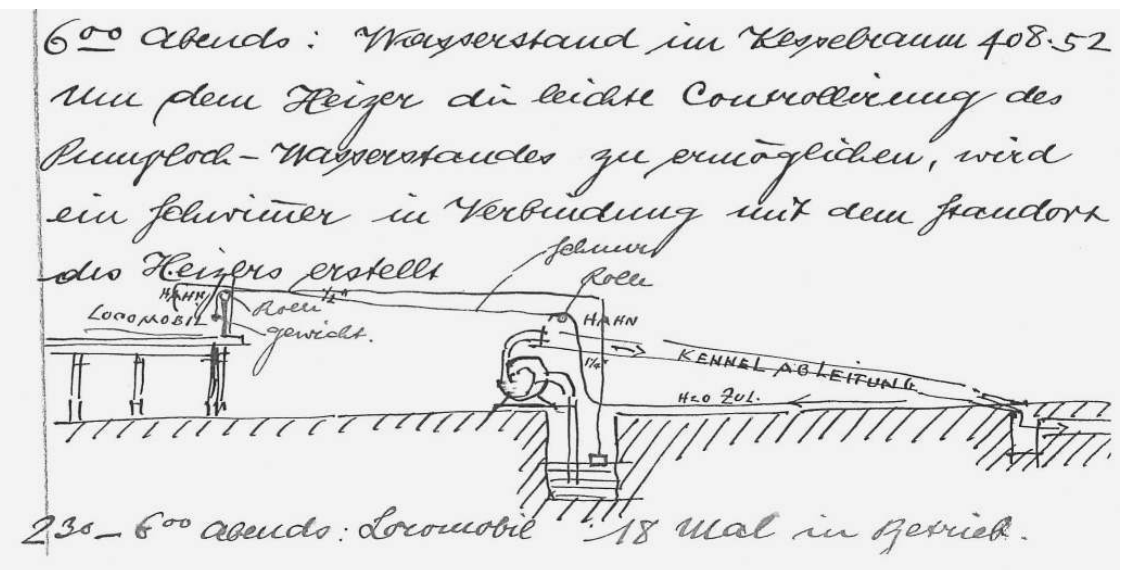

Fig. 6. Croquis sur l'installation d'une pompe centrifugeuse et d'une machine à vapeur sur le chantier de l'immeuble de bureaux 'Fraumünsteramt', Zürich, 1899. (Archives municipales de Zurich, V.G.b.253:2-4, p. 39).

cette profession ${ }^{39}$. Il peut sembler étrange qu'il n'opte pas pour une machine à vapeur après avoir constaté les difficultés de pompage. Cela peut soit s'expliquer par le coût élevé de celles-ci, soit par les lacunes de la formation des architectes de la seconde partie du XIX ${ }^{\mathrm{e}}$ siècle dans le domaine mécanique. De ce fait, leur savoir quant au fonctionnement des machines à vapeur, au choix d'une machine et aux possibles améliorations et adaptations de ces machines en fonction des chantiers reste faible.

En fin de compte, l'option prise par Gull concernant la pompe à jets d'eau („Wasserstrahlpumpe“) se révèle peu pertinente; après cinq jours d'un usage continu, le niveau d'eau reste toujours aussi haut du fait de la forte pression de l'eau souterraine. C'est finalement le propriétaire de l'entreprise de maçonnerie, fournissant les ouvriers aux autorités, qui propose une solution. Il recommande une machine à vapeur de quinze chevaux en association avec une pompe centrifuge de 150 millimètres. «Après avoir considéré à nouveau l'usage des pompes à aspiration, M. Lauffer recommande dans l'après-midi l'usage d'une machine à vapeur. J'ai parlé de cette suggestion à M. Gull [...] qui approuve» (fig. 6) ${ }^{40}$. Le fait qu'il délivre ses conseils si tardivement peut s'expliquer par ses profits réalisés en fournissant la main-d'œuvre nécessaire aux précédentes tentatives. Quoi qu'il en soit, ses recommandations apparaissent logiques : une pompe centrifuge associée à une machine à vapeur est alors la solution la plus rationnelle et efficace dans cette situation ${ }^{41}$. Les

39. Voir par exemple Andrew Saint, Architect and Engineer. A Study in Sibling Rivalry, New Haven/London, Yale University Press, 2007.

40. „Nachdem zuerst der einfache Handpumpenbetreib in Aussicht genommen war, empfiehlt am Nachmittag Herr Lauffer ein Locomobil [...] herzunehmen. Ich bespreche diesen Vorschlag [...] mit Herrn Gull, welcher demselben beipflichtet." AdZ, V.G.b.253. : 2-4, notation du 7 avril 1899.

41. Voir lemme „Pumpen“, paragraphe „Zentrifugalpumpen“ in: Otto Lueger, Lexikon der gesamten Technik... op. cit., volume 7, p. 621-623. 
précisions techniques qu'il donne pour la machine de "quinze chevaux" et la pompe centrifuge de " 150 millimètres» indiquent qu'il propose une machine puissante pour un tel chantier ${ }^{42}$. Le but est finalement atteint et le site est rapidement asséché.

Ce cas peut être questionné dans son ensemble plus avant pour comprendre quels savoirs étaient attendus au sein de chaque groupe professionnel. Les ouvrages concernant la supervision des sites - qui peuvent être considérés comme représentatifs du savoir spécifique des superviseurs des travaux ou des autres acteurs dont l'activité est essentiellement pratique - recommandent deux alternatives ${ }^{43}$ : soit une pompe aspirante actionnée à la main, soit une pompe centrifuge associée à une machine à vapeur. Ces ouvrages offrent aussi un moyen de décider de la meilleure solution selon les cas rencontrés. Deux exemples de calcul à réaliser sont présentés. Le lecteur peut ainsi utiliser une méthode quantitativement fondée pour prendre une décision pratique. Tous les acteurs mentionnés sont susceptibles d'utiliser de tels ouvrages - comme le très diffusé Schweizer Bau- \& Ingenieur-Kalender - pour faire une grossière estimation des coûts. Il apparaît pourtant que dans ce cas d'espèce, aucun d'entre eux n'a eu recours à ces ouvrages. S'ils l'avaient fait, ils auraient pu voir que le coût était sensiblement le même pour les trois options : les pompes aspirantes et les pompes à jets d'eau ${ }^{44}$ ont les mêmes coûts opérationnels et la machine à vapeur coûte, elle, à peine moins $\operatorname{cher}^{45}$ malgré les coûts d'installation et de démontage des machines ${ }^{46}$. Cette approche qui consiste à anticiper les coûts des différentes installations ne semble mobilisée que par le propriétaire et l'entreprise de maçonnerie, ce qui n'est pas surprenant. Être le propriétaire d'une entreprise prospère appelle une expertise dans l'évaluation des coûts et la définition des prix, deux caractéristiques de ces entrepreneurs.

L'analyse des détails d'une controverse attachée au choix de pompes permet de tirer plusieurs conclusions ${ }^{47}$. Tout d'abord, les protagonistes sur le chantier arbitrent leur décision à partir de l'usage concret des machines sur le site. Leur choix est nécessairement lié, sans pour autant s’y réduire, aux usages possibles des machines. Ensuite, les différents groupes sociaux ne favo-

42. Pour comparer ce qui était disponible : Schweizerischer Bau-und Ingenieur-Kalender, volume 2, Zürich, Cäsar Schmidt, 1902, p. 75.

43. Voir Gustav Tolkmitt, Bauaufsicht... op. cit., p. 129-131.

44. Le coût journalier d'une opération de pompage peut être évalué à 130 francs suisse, cf. Schweizerischer Bau- und Ingenieur-Kalender, volume 1, Zürich, Cäsar Schmidt, 1900, p. 50. Il n'existe pas d'archives des prix de location des pompes à jets d'eau. Cependant, les manuels d'époque permettent d'établir que les coûts de mise en œuvre sont les mêmes que pour les pompes aspirantes manœuvrées manuellement. L. Franzius et F. Lincke (ed.), Die Baumaschinen... op. cit., volume 1, (1 ère édition 1885) 1897, p. 103. 45. Le tarif de location pour les machines à vapeur avec des pompes, en excluant le combustible, est d'environ 35 francs suisse par jour; voir Schweizerischer Bau-und Ingenieur-Kalender, volume 1, Zürich, Cäsar Schmidt, 1900, p. 50. Le coût du travail est donné dans le Schweizerischer Bau- und IngenieurKalender seulement depuis 1902, mais les variations sont faibles avec les années précédentes. Un machiniste et le combustible pour faire fonctionner la machine coûte, selon les autorités, autour de 75 francs suisse; cf. Schweizerischer Bau-und Ingenieur-Kalender, volume 2, Zürich, Cäsar Schmidt, 1902, p. 75. 46. Voir Schweizerischer Bau- und Ingenieur-Kalender, volume 1, Zürich, Cäsar Schmidt, 1900, p. 50.

47. Mon travail porte par ailleurs sur un plus large panel de cas. 
risent pas les mêmes machines pour la même tâche, et ces choix sont à mettre en relation avec leur position sociale et le type de formation qu'ils ont reçue. Enfin, l'entrepreneur joue un rôle décisif dans l'intégration sur le site de nouvelles machines de construction.

Ces conclusions permettent de formuler quelques hypothèses sur la dimension sociale des techniques. D'une part, le pouvoir des groupes sociaux et de leurs représentants détermine le choix de l'usage des produits techniques $^{48}$. Et le pouvoir social n'étant pas réparti de façon uniforme, un processus d'intégration technique peut être suspendu ou détruit par le jeu des forces sociales. D'autre part, si nous considérons l'entrepreneur comme un personnage-clé dans les processus d'intégration de la nouveauté technique, nous devons nous interroger sur le sens de cette position d'expert, largement questionnée par de récents travaux ${ }^{49}$. Tous les protagonistes sur le site maîtrisent un corps de connaissances spécifiques et reconnues, mais ce qui place l'entrepreneur à part et lui donne un statut d'expert, c'est sa longue expérience. Paradoxalement, malgré l'importance grandissante du savoir académique sur les chantiers à la fin du XIX siècle, l'expérience demeure essentielle dans la prise en charge et l'intégration des nouveautés techniques. Au final, malgré les récentes mises en cause de cette lecture ${ }^{50}$, on peut souligner que les technologies nouvelles arrivent dans le bâtiment dans des espaces socialement divisés et constitués de longue date. De nombreux travaux sur l'histoire de la construction ont souligné le rôle central joué par les entreprises nouvellement spécialisées dans l'adoption de techniques nouvelles ${ }^{51}$. Cependant, comme le montre une étude de cas précise, les nouveaux objets techniques ne chamboulent pas l'ordre social, loin s'en faut. Bien plus, ce sont les cadres sociaux qui déterminent l'intégration des techniques et plus encore qui modèlent les objets techniques.

\section{POUVOIRS ET INTÉGRATION DES TECHNIQUES}

Afin de montrer comment la technologie peut façonner et recomposer les rapports sociaux, on doit en revenir au terrain de la pratique. Les machines à vapeur ont besoin d'être constamment approvisionnées en eau et en énergie. C'est une tâche spécifique que les ouvriers du bâtiment ne sont pas ordinaire-

48. Klein and Kleinmann soulignent aussi ce point : Hans K. Klein et Daniel Lee Kleinmann (eds.), 'The Social Construction..., op. cit., p. 34-35.

49. Eric H. Ash, "Introduction : Expertise and the Early Modern State", in Eric H. Ash, Expertise. Practical Knowledge and the Early Modern State, Osiris 25, Chicago, University of Chicago Press, 2010, p. 1-24, ici p. 4-11.

50. Hans K. Klein et Daniel Lee Kleinmann, 'The Social Construction... op. cit., p. 36-38.

51. Christopher Powell, "Who did what: Division of labour among construction-related firms", in Santiago Huerta (ed.), International Congress on Construction History, Proceedings of the First International Congress on Construction History: Madrid, 20th-24th January 2003, Madrid, Juan de Herrera, 2003, p. 1649-1655, ici p. 1654-1655. 
ment aptes à faire et qu'ils considèrent comme ne relevant pas de leurs fonctions. De ce fait, un mécanicien doit nécessairement être embauché. Loin d'être un changement négligeable; il s'agit d'une transformation profonde de l'organisation sociale des chantiers. Contrairement aux artisans traditionnels, les mécaniciens peuvent être considérés comme des cols bleus qualifiés - un groupe social inattendu ici car traditionnellement absent des chantiers de construction. Par conséquent, le recours à de nouvelles machines impose l'apparition d'un groupe professionnel qui n'avait jusqu'alors pas sa place sur le chantier.

Plus encore, l'intégration de ce nouveau groupe de travailleurs est liée aux changements dans les responsabilités sur les chantiers. Habituellement, la supervision et le contrôle des travaux finis étaient de la responsabilité du superviseur du site. Cependant, sur la question de la mise en œuvre et de la direction des machines à vapeur, le superviseur du site n'avait alors que très peu d'éléments de savoir, que ni sa formation académique au Technika, ni les manuels de supervision des sites ne délivraient. Les notes prises pour la construction du bâtiment Fraumünsteramt montrent clairement combien les connaissances du superviseur étaient maigres en la matière ${ }^{52}$. Il était pourtant nécessaire d'instruire, de favoriser l'intégration, de superviser et de vérifier le travail du mécanicien. Dans le cas présent, il n'existait qu'une seule solution: que l'entrepreneur de travaux s'en charge. Sur le chantier du Fraumünsteramt, le déplacement de responsabilités apparaît informel : si techniquement le superviseur est en charge de ces questions, il est en pratique largement influencé par le maitre d'ouvrage. Des efforts sont cependant réalisés pour formaliser ce changement : le conducteur de travaux Kruck propose de préciser ce partage des tâches dans les contrats à venir touchant à la construction des bâtiments civils ${ }^{53}$.

Une fois encore, l'incident décrit peut paraitre limité mais il est à l'image de quantité d'autres incidents qui indiquent une tendance plus large : le mécanicien fait figure de nouveau venu, dont les racines sociales n'étaient alors pas ancrées dans les traditions des chantiers comme l'étaient celles des corporations de maçons, de couvreurs ou de charpentiers. Les développements techniques, telles que les nouvelles techniques de construction comme le béton armé, les systèmes sanitaires complexes ou les nouvelles machines de construction, ont pour effet d'introduire de nouveaux groupes sociaux sur les chantiers ${ }^{54}$. Si parfois ces nouveaux acteurs peuvent s'intégrer avec facilité dans les processus de construction, ils demeurent le plus souvent en rupture du fait d'une culture professionnelle différente. Leurs aptitudes peuvent être considérées comme externes et de ce fait ils sont contraints de porter

52. „Installieren der Pumpe unter Behilfe von Lauffer \& Franceschetti“, AdZ, V.G.b.253.:2-4, notation du 15 avril 1899.

53. AdZ, V.G.b.253. : 2-4, notation du 25 avril 1899

54. Christoph Rauhut, Die Baustelle um 1900... op. cit. 
la responsabilité de leur travail en dehors d'un groupe constitué. L'effet de cette évolution a été, par exemple, qu'ils continuent de travailler durant les congés et les jours fériés tels que la fête du travail ${ }^{55}$. De façon plus significative encore, ils signent de nombreux contrats avec les autorités et sont plus clairement intégrés dans les décisions relevant de l'urbanisme. Au regard des usages des machines de construction, ce développement se met réellement en place au début du XXe siècle mais le phénomène s'enracine manifestement

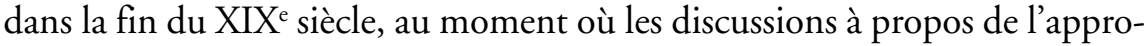
priation de l'usage des machines de construction sur les chantiers deviennent quotidiennes.

\author{
Christoph Rauhut est doctorant à l'Institut \\ für Denkmalpflege und Bauforschung, IDB (Zürich, Suisse) \\ Traduit de l'anglais par M. Charpy et F. Jarrige.
}

55. Christoph Rauhut, „'denn praktisch zu sein, liegt im Streben unserer Zeit' - Rationalisierungsbestrebungen auf der Baustelle um 1900“... op. cit. 